\title{
Plasma ST6Gal1 is Dispensable for IgG Sialylation
}

\author{
Douglas M Oswald ${ }^{1}$, Sylvain D Lehoux ${ }^{2}$, Julie Y Zhou ${ }^{1}$, \\ Leandre M Glendenning ${ }^{1}$, Richard D Cummings ${ }^{2}$, Brian A Cobb ${ }^{1,3}$
}

${ }^{1}$ Case Western Reserve University School of Medicine, Department of Pathology, Cleveland, OH, USA

${ }^{2}$ Beth Israel Deaconess Medical Center, Harvard Medical School Center for Glycoscience, National Center for Functional Glycomics, Boston, MA, USA

${ }^{3}$ Corresponding author

Correspondence: Brian A Cobb, PhD; brian.cobb@case.edu; 10900 Euclid Avenue, Cleveland, OH 44106; Phone 216-368-1263; Fax 216-368-0494

Running Head: Hepatocyte ST6Gal1 does not sialylate IgG

Keywords: ST6Gal1, glycobiology, IgG, sialylation, hepatocyte, plasma, sialic acid 


\section{Abstract}

2 The glycosylation of IgG has attracted increased attention due to the impact of N-glycan

3 modifications at $\mathrm{N} 297$ on IgG function, acting primarily through modulation of Fc domain

4 conformation and Fc $\gamma$ receptor binding affinities and signaling. However, the mechanisms

5 regulating $\operatorname{IgG}$ glycosylation and especially $\alpha 2,6$-sialylation of its $\mathrm{N}$-glycan remain poorly

6 understood. We observed previously that IgG is normally sialylated in mice with B cells lacking

7 the sialyltransferase ST6Gal1. This supported the hypothesis that IgG may be sialylated outside of

8 B cells, perhaps through the action of hepatocyte-released plasma ST6Gall. Here we demonstrate

9 that this model is incorrect. Animals lacking hepatocyte expressed ST6Gall retain normal IgG

$10 \alpha 2,6$-sialylation, despite the lack of detectable ST6Gal1 in plasma. Moreover, we confirmed that

11 B cells were not a redundant source of $\operatorname{IgG}$ sialylation. Thus, while $\alpha 2,6$-sialylation is lacking in

12 IgG from mice with germline ablation of ST6Gal1, IgG $\alpha 2,6$-sialylation is normal in mice lacking

13 ST6Gal1 in either hepatocytes or B cells. These results indicate that $\operatorname{IgG} \alpha 2,6$-sialylation arises

14 after release from a B cell, but is not dependent on plasma-localized ST6Gall activity. 


\section{Introduction}

Research beginning in the early 1980s has firmly established the links between changing

17 biological and disease states with alterations in IgG glycosylation (reviewed in (1)). Human and

18 mouse IgG contains a single and conserved N-linked glycan at N297 of the Fc domain in each

19 heavy chain, which is primarily a biantennary complex N-glycan varying in of GlcNAc bisection,

20 galactosylation, core fucosylation, and terminal $\alpha 2,6$-linked sialylation (2). A number of early

21 studies characterized IgG glycosylation in the context of rheumatoid arthritis (RA) and reported a

22 decrease of galactosylation and sialylation associated with disease. Similar studies involving a

23 variety of inflammatory conditions have extended these findings to establish that inflammatory

24 diseases lead to a decrease of galactosylation and sialylation, directly demonstrating that these

25 modifications to the IgG glycan are under regulatory control.

The importance of sialylation of IgG was documented first in 2006, when it was reported

27 that the active portion of high dose intravenous immunoglobulin therapy (IVIg) responsible for its

28 anti-inflammatory properties in autoimmune patients was the $\alpha 2,6$-sialylated glycoforms of IgG

29 (3). Subsequent studies have largely documented the linkage of IgG glycosylation changes to

30 various physiological and pathological states including pregnancy $(4,5)$, tuberculosis (6), HIV

$31(7,8)$, diabetes (9), kidney dysfunction (10), lupus (11), and others, as has been recently reviewed

32 (1). Mechanistic work has focused on how sialylated IgG reduces inflammation (12,13), but many

33 outstanding questions remain $(14,15)$.

34 Our recent work has established that the $\alpha 2,6$-sialylation of $\operatorname{IgG}$ is independent of the

35 required enzyme ST6Gall in B cells (16), using a novel B cell-specific conditional knockout of

36 ST6Gal1 (BcKO). Despite lacking ST6Gal1, these BcKO animals express $\alpha 2,6$-sialylated IgG at

37 levels indistinguishable from wild type (WT) mice. Although there are published data on the 
relationship of B cell ST6Gall expression and IgG sialylation (17), as well as other models of B cell glycan modification (18), it is clear that B cell ST6Gal1 is not required for IgG sialylation (16). Instead, the observations with the BcKO mouse point to a previously suggested possibility (19),

41 based on a growing body of work, that the complex N-glycans of $\operatorname{IgG}$ and other circulatory 42 glycoproteins can be modified after they are secreted into the fluid phases of the body - a process 43 called extracellular sialylation.

ST6Gal1 is highly and inducibly expressed in hepatocytes as a membrane-bound enzyme

45 (20); however, due to action by the protease BACE-1, a soluble form of ST6Gall can be released 46 into plasma (21,22), where ST6Gal1 retains enzymatic activity (16,23). Coupling our BcKO 47 observations (16) with previous findings linking liver ST6Gal1 with IgG sialylation (16,19), we

48 and others hypothesized that the liver-released ST6Gal1 may be required for the synthesis of 49 sialylated IgG. Indeed, the development of mice lacking the liver-specific P1 region of the 50 ST6Gal1 promoter $(\Delta \mathrm{P} 1)$ was reported to have both reduced circulatory ST6Gal1 activity (23) and

51 reduced IgG sialylation (19). Consistent with this model, we showed that ST6Gall expression in

52 the liver is dramatically increased in halos surrounding central veins where hepatocyte proteins

53 and glycoproteins are released into circulation. Finally, there is evidence that human platelet

54 granules may supply the nucleotide-activated sugars that serve as donors for glycosyltransferases

55 within the plasma (24), and our prior findings confirm that murine platelets can also supply CMP-

56 sialic acid for ST6Gal1 activity (16).

However, to conclusively establish whether extracellular sialylation from plasma-localized

58 ST6Gal1 may be contributing to $\alpha 2,6$-sialylation of IgG, we generated a hepatocyte-specific

59 conditional knockout of ST6Gall (HcKO) using the albumin-Cre mouse, which we have recently

60 reported $(25,26)$. We observed, as predicted, a complete ablation of $\alpha 2,6$-linked sialic acids on 
61 liver-produced circulatory glycoproteins and hepatocyte cell surfaces, but not in other cells or

62 tissues. Here, we describe the impact of hepatocyte ablation of ST6Gall on IgG sialylation. Our

63 results indicate that despite the loss of detectable plasma ST6Gall in the HcKO mice, IgG

64 sialylation was unchanged compared to WT controls. Additionally, BACE-1 knockout mice also

65 had normal IgG sialylation. Importantly, mice with germ-line loss of ST6Gal1 lacked sialylated

66 IgG, thereby confirming the absolute requirement for ST6Gall in IgG sialylation. We further

67 developed a mouse lacking ST6Gall in both the hepatocyte and B cell compartments (BHcKO) to

68 determine whether each cell might be able to compensate for the other. IgG sialylation remained

69 unchanged. These findings demonstrate that neither ST6Gal1 in plasma nor in B cells generates

70 IgG sialylation, and suggest that an unidentified compartment outside of these must be necessary

71 to regulate the sialylation of $\mathrm{IgG}$. 


\section{Results}

Total plasma glycoprotein glycosylation in WT and HcKO mice was characterized with

74 and without ovalbumin in alum immunization using a plate-based lectin ELISA assay as we have

75 previously described (27). We found that regardless of immunization status, SNA signal was very

76 low in HcKO samples (Fig 1A). Interestingly, MAL-II, WGA, and PHA-E all showed marked

77 increases in naïve $\mathrm{HcKO}$ mice compared to WT, but those differences and overall levels were

78 dramatically reduced in both strains upon immunization, despite that the immunized plasma

79 samples were taken 6 weeks after the final boost to allow the mice to return to baseline. Conversely,

80 immunization increased LCA and AAL, both fucose-detecting lectins (Fig 1A). Principal

81 component analysis (PCA) of these data illustrated the major differences between naïve WT and

82 HcKO plasma glycosylation (Fig 1B). Immunization significantly reduced the difference between

83 strains, but the immunized cohort was significantly different from their naïve counterparts (Fig

84 1B), with the exception that SNA-detectable $\alpha 2,6$-sialylation remained distinct between strains

85 (Fig 1A).

Next, the IgG response to immunization was quantified. We found that both WT and HcKO

87 mice strongly responded to immunization, although immunized WT mice did responded stronger

88 (Fig 2A). SNA and anti-Fc antibody ELISA on purified IgG from each immunized strain revealed

89 no detectable difference in total sialylation between strains (Fig 2B). The dramatically increased

90 presence of IgG might explain the decreases in SNA and ConA, and increased LCA signal in the

91 total plasma samples of immunized mice (Fig 1A) due to the low sialylation and high fucosylation

92 characteristics of typical IgG N-glycans (2).

93 To more robustly quantify $\alpha 2,6$-sialylation, we performed mass spectrometry analysis on

94 IgG glycans using 4-(4,6-dimethoxy-1,3,5-triazin-2yl)-4-methylmorpholinium chloride (DMT- 
$95 \mathrm{MM}$ ) activation of the sialic acid carboxylate group, which upon permethylation leads to selective amidation of $\alpha 2,6$-linkages and spontaneous lactonization of $\alpha 2,3$-linkages that are easily

97 differentiated by mass spectrometry due to an added +13 Da on $\alpha 2,3$-linked sialic acids (28). The

98 degree of sialylation was found to be approximately $20 \%$ in WT mice, but over $30 \%$ in $\mathrm{HcKO}$

99 mice (Fig 2C). We found that the majority of sialic acids in murine IgG are present in an $\alpha 2,3$

100 linkage, whereas $\alpha 2,6$ linkages were a minority, although there was no significant difference

101 between strains (Fig 2D), consistent with prior studies (2). A detailed breakdown of sialylated N-

102 glycans further reveals that the dominant structures were G2S1 with either Neu5Ac or Neu5Gc,

103 and plus or minus core fucose (Fig 2E and 2F). Very few di-sialylated N-glycans were present in

104 either strain on IgG at this time point (i.e. 2 weeks post final boost immunization).

105 BACE-1 has been shown to be necessary for ST6Gal1 release from the liver (22). Thus,

106 the BACE-1 knockout mouse would be expected to phenocopy HcKO mice in terms of $\operatorname{IgG}$

107 sialylation due to a lack of circulatory ST6Gal1. We obtained plasma from naïve WT and BACE-

1081 knockout mice from the Jackson Laboratories. IgG concentration was higher in the unimmunized

109 BACE-1 knockout mice (Fig 3A), and the degree of IgG $\alpha 2,6$-sialylation, as measured by SNA

110 binding, was indistinguishable from WT mice from the same facility (Fig 3B). Finally, we

111 confirmed that ST6Gal1 was indeed required for IgG $\alpha 2,6$-sialylation using the germline ST6Gal1

112 knockout mouse. IgG from these mice lacked SNA-detectable $\alpha 2,6$-sialylation (Fig 3C).

113 These data demonstrate that hepatocyte-released ST6Gall is dispensable for IgG

114 sialylation, suggesting two possibilities. One is that either B cells and hepatocytes (i.e. circulatory

115 ST6Gal1) are redundant pathways and thus can compensate for each other, yielding a lack of IgG

116 sialylation differences in each of the $\mathrm{HcKO}$ and $\mathrm{BcKO}$ mouse models. The other is that neither of

117 these compartments are necessary for IgG sialylation. In order to differentiate between these two 
118 possibilities, we created a mouse in which ST6Gal1 was ablated in both hepatocytes and B cells

119 (BHcKO; Fig 4A). We first confirmed the lack of hepatocyte $\alpha 2,6$-sialylation using confocal

120 microscopy and found a similar pattern of SNA staining in the BHcKO liver (Fig 4B) as we

121 previously reported for the HcKO mouse $(25,26)$. Likewise, B cells were analyzed by flow

122 cytometry with SNA and ECL, and we found the same decrease in SNA and increase in ECL (Fig

$1234 \mathrm{C}$ ) as we previously reported for the BcKO mouse (16). These data confirm that the ablation of

124 ST6Gall was found in both target tissues as expected.

125 Before analyzing the IgG itself, we also confirmed that the HcKO and BHcKO mice lacked

126 ST6Gall activity in the plasma using a plate assay as we have previously described (27). We found

127 that both the WT and BcKO strains showed strong ST6Gall activity in plasma samples when

128 supplied with exogenous CMP-sialic acid, but neither the HcKO nor BHcKO mice showed

129 detectable activity (Fig 5A). Likewise, we confirmed that plasma-localized ST6Gal1 retains

130 activity in vivo by injecting CMP-sialic acid via the tail vein and monitoring for IgG sialylation.

131 Although the total IgG concentration did not change, the degree of IgG sialylation increased (Fig

132 5B), suggesting that the enzyme in circulation can function if given adequate donor, but that it is

133 dispensable in maintaining IgG sialylation with or without immunization.

134 Next, we analyzed the overall IgG titer (Fig 6A) and IgG subclass distribution (Fig 6B) in

135 the WT, BcKO, HcKO, and $\mathrm{BHcKO}$ strains with and without immunization. In contrast to the data

136 in Figure 2 in which IgG N-glycans were analyzed 2 weeks after the final immunization booster,

137 we chose to perform the analysis at 8 weeks after the last injection to ensure all animals had

138 returned to homeostasis following immune activation. In general, all three knockout strains had a

139 slightly reduced $\mathrm{IgG}$ concentration following immunization. With the exception of the $\mathrm{BcKO}, \mathrm{IgG}$

140 subclass distribution was also not substantially different from WT. The BcKO showed an 
141 expansion of IgG2a and IgG2b with a concomitant decrease in IgG1. More importantly, we found

142 using mass spectrometry that the degree of sialylation and the relative distribution of sialic acid

143 linkages was indistinguishable among all four strains (Fig 6C and 6D). A further breakdown of the

144 dominant sialylated N-glycans revealed similar results to the WT and HcKO mice at two weeks in 145 which singly sialylated species dominated (Fig 2), although there was an increase in dual sialylated 146 species at this later time point (compare Fig 2E and 6E). These findings suggest that sialylation 147 increases over time following a return to homeostasis (Fig 6E and 6F). These data reveal that both 148 circulatory/hepatocyte as well as B cell ST6Gall are dispensable and do not compensate for each 149 other.

Finally, the mass spectrometry data at 2 and 8 weeks post final immunization booster

151 seemed to indicate a progressive sialylation process during the return to homeostasis after

152 immunization/exposure (compare Fig $2 \mathrm{~F}$ and $6 \mathrm{~F}$ ). Thus, we first analyzed each of the main

153 sialylated species independently based on the distribution of $\alpha 2,3$ and $\alpha 2,6$ linkages. Among the

154 mono-sialylated N-glycans, we found that there are shifts in the linkage distribution at different

155 time points, but that this appears to be influenced by the strain (Fig 7A). For example, in the

156 G2S1 N-glycan containing Neu5Ac, both WT and HcKO strains showed low initial $\alpha 2,6$

157 sialylation at 2 weeks, but that increased over time to yield equivalent $\alpha 2,3$ and $\alpha 2,6$

158 distributions by week 8. The equivalent distribution was not seen in $\mathrm{BcKO}$ or $\mathrm{BHcKO}$ mice at 8

159 weeks. Similarly, among the di-sialylated species, there was some change in the distributions,

160 depending on the strain (Fig 7B). In general, we found that the relative amount of $\alpha 2,6$ linked

161 sialic acids increased over time, and this correlated with an increase in di-sialylated N-glycans

162 with increasing time after the last immunization in both WT and HcKO mice (Fig 8). 


\section{Discussion}

Here we demonstrate, as might be expected, that $\alpha 2,6$-sialylation of IgG by ST6Gall arises

165 by the action of ST6Gal1, as documented using germ-line deletion of ST6Gal1. Unexpectedly our

166 prior studies had shown that such sialylation of IgG does not arise by the action of ST6Gal1 in B

167 cells, although the loss of B cell ST6Gal1 led to a loss of cell surface $\alpha 2,6$-sialylation and

168 replacement by terminal galactose (16). These prior studies led us to test the hypothesis that $\operatorname{IgG}$

$169 \alpha 2,6$-sialylation might arise through the action of liver-derived soluble ST6Gall in the plasma.

170 However, our results demonstrate that neither the ST6Gall in plasma or in B cells is chiefly

171 responsible for $\operatorname{IgG} \alpha 2,6$-sialylation.

The plasma-derived ST6Gal1 is known to be released from the liver through the action of

173 the protease BACE-1 (21,22). The notion that IgG sialylation might involve plasma ST6Gal1

174 originated in studies utilizing a mouse with the P1 region of the ST6Gal1 promoter ablated (23).

175 The $\Delta \mathrm{P} 1$ mouse has a documented reduction, albeit not an ablation, of plasma ST6Gal1 activity

176 along with a reduction in $\operatorname{IgG}$ sialylation $(19,23)$. We considered that the creation of the $\mathrm{BcKO}$

177 mouse and the observation that IgG sialylation was unchanged (19) seemed to support this model.

178 Through the creation of the combined HcKO and BHcKO mice, our results here demonstrate that

179 plasma ST6Gal1 is not necessary for IgG sialylation. Complete loss of plasma ST6Gall activity,

180 ablation of BACE-1, and a combination knockout in hepatocytes and B cells collectively

181 demonstrate that IgG sialylation occurs elsewhere, but certainly not in either B cells nor plasma.

182 The lack of plasma ST6Gal1 making an impact on glycans within the circulation is further

183 supported by the fate of the B cells in the BcKO mouse (19). These cells have a dramatically

184 reduced surface $\alpha 2,6$-sialylation, yet are in direct contact with ST6Gal1 in plasma just like IgG.

185 We were puzzled by the apparent contradiction, that whereas B cell surfaces lacked $\alpha 2,6-$ 
186 sialylation, their secreted IgG was 2,6-sialylated. The current HcKO mouse supports the

187 interpretation that plasma ST6Gal1, while functional, cannot functionally sialylate B cells lacking

188 endogenous ST6Gal1. This is most likely due to the lack of CMP-sialic acid required as a donor

189 for the sialylation reaction under most conditions.

Another key uncertainty has been why the $\Delta \mathrm{P} 1$ mouse does not phenocopy the HcKO strain.

191 The original description of the $\Delta \mathrm{P} 1$ mouse exhibited a modest reduction in circulatory ST6Gal1

192 protein and activity using in vitro assays, likely due to the fact that expression of the enzyme is

193 incompletely knocked down. Moreover, since the creation of the $\Delta \mathrm{P} 1$ mouse, it has become clear

194 that these animals carry interesting hematopoietic phenotypes (29-31) that are also not

195 phenocopied in the HcKO mice $(25,26)$. We reason that the manipulation of the P1 region of the

196 promoter has an impact beyond the liver and circulatory activity of ST6Gal1, and that it is likely

197 involved with expression of ST6Gall in multiple hematopoietic cell lineages. Given that the HcKO

198 mouse lacks detectable ST6Gal1 activity in plasma, we believe that non-liver reductions in

199 expression are the source of the phenotypes described using the $\Delta \mathrm{P} 1$ mouse, and not changes in

200 plasma ST6Gall.

201 Beyond the continuing puzzle regarding the non-plasma and non-B cell location of $\operatorname{IgG}$

202 sialylation, another observation herein is the apparent high proportion of $\alpha 2,3$-linkages among

203 sialylated $\mathrm{N}$-glycans found on IgG in these mice. Work citing $\alpha 2,3$ sialylation of IgG is extremely

204 limited, although $\alpha 2,3$ - and $\alpha 2,6$-sialylation of murine IgG was recently documented, as well as

205 strain differences. Unfortunately, a great number of studies on IgG glycosylation does not address

206 the specific nature of sialic acid linkages. As such, much of the discussion and analysis of IgG

207 sialylation may be, to some degree, problematic. The effects of $\alpha 2,3-$ vs $\alpha 2,6$-sialylation on the 208 activities of IgG are not well documented. Thus, it is likewise not clear whether $\alpha 2,3$ sialylation of 
209 IgG affects its affinity to the Fc $\gamma$ receptor in the same way as $\alpha 2,6$-sialylation, nor is it known how

$210 \alpha 2,3$-sialylation might impact IgG structure. In this regard, while IgG glycosylation invariably

211 occurs on the Fc portion at N297, it is known that additional N-glycans might be present on a

212 subset of IgG through N-glycosylation of the Fab domain. However, this would not affect our

213 overall interpretation, as we analyzed total N-glycans and their total sialylation, in which $\alpha 2,6-$

214 sialylation could of course impact both types of N-glycans.

215 Finally, we found that the sialylation of IgG N-glycans changes as a function of distance

216 in time from the most recent exposure or immunization. There have been proposals as to the

217 possibility of programming the glycoform of specific clones of antibodies in vivo via vaccination

218 (32), but our data suggest that IgG glycosylation is perhaps not stable enough for this to work as a

219 therapeutic approach, at least in the murine system, since the degree of sialylation as well as the 220 proportion of singly or doubly sialylated species changes longitudinally after immunization.

221 In summary, our data now allows us to reject the model that IgG sialylation occurs within

222 the circulatory microenvironment through plasma-localized ST6Gal1, as well as documenting a

223 significant degree of $\alpha 2,3$ sialylation on IgG N-glycans in mice, and an unstable degree of

224 sialylation over time following immunization. The findings point to an unidentified anatomical

225 location or cell type in which IgG is sialylated. A leading candidate may be platelets, given their

226 expression of ST6Gal1 and demonstrated ability to release CMP-sialic acid $(16,24)$, yet much more

227 research is needed to clarify these mechanisms in vivo. 


\section{Experimental procedures}

229 Animal care and use

All animal work was approved by the institutional animal care and use committee (IACUC)

231 of Case Western Reserve University. Mice were procured from Jackson Laboratories and kept/bred

232 in the CWRU ARC in accordance with IACUC guidelines. ST6Gal1 ${ }^{\mathrm{f} / \mathrm{f}}$ mice (stock 006901) and

233 Alb-Cre mice (stock 003574) were crossed to produce the HcKO line, as we document in our

234 previous manuscript (26). Wherever the phrases "wild type" or "parental" are used, they refer to

235 mice of the ST6Gal1 ${ }^{\mathrm{f} / \mathrm{f}}$ background, which carry no phenotype, with the exception of the control

236 mice for the BACE-1 study which were C57B1/6J mice directly housed by Jackson Laboratories

237 (JAX). In that case, the WT and BACE-1 knockout mouse serum was harvested by and purchased

238 directly from JAX. BcKO mice were produced by crossing the ST6Gal1 ${ }^{\mathrm{f} / \mathrm{f}}$ mice with CD19-Cre

239 mice (stock 006785), and were used as experimental when homozygous on the allele and

240 heterozygous on the CD19-Cre allele, as described (16). BHcKO mice were combined by crossing

241 homozygous breeder of the BcKO and HcKO line, resulting in ST6Gal1 ${ }^{\mathrm{f} / \mathrm{f}}, \mathrm{Alb}-\mathrm{Cre}^{+/-}, \mathrm{CD} 19-\mathrm{Cre}^{+/-}$

242 mice.

\section{Animal harvest}

244 For harvest, mice were euthanized using $\mathrm{CO}_{2}$ following standard procedures. Blood was

245 drawn via cardiac puncture. Plasma was separated from blood by spinning at $2000 \times \mathrm{g}$ for 15

246 minutes, and aspirating the liquid phase.

Flow cytometry

Flow cytometry was performed on cells isolated freshly ex vivo from mice, as described

249 elsewhere (16). Liver lobes were diced and digested in $2.5 \mathrm{mg} / \mathrm{ml}$ collagenase (cat) in $3 \%$ FBS

250 for 45 minutes at $37^{\circ} \mathrm{C}$. Tissues were diced and mashed through 70 um nylon filters in $10 \mathrm{ml}$ of 
251 PBS. Cells were spun down, then RBC lysis was performed using pharmlyze (BD Biosciences,

252 555899) at $4{ }^{\circ} \mathrm{C}$ for 5 minutes, then quenched with PBS. Cells were blocked for 30 minutes in

253 Carbohydrate Free Blocking Solution (Vector) and stained with the following reagents: SNA-FITC

254 (Vector), CD19-APC (Biolegend). Flow cytometry was run on an Accuri C7. Data was analyzed 255 using FlowJo.

256 Histology

257 Histology embedding and sectioning was performed by the CWRU Cancer Center 258 Histology Services Core as previously described (16). Tissues for histological analysis were 259 resected from mice and fixed in 10\% formalin solution. Tissue blocks were embedded in paraffin 260 wax, sectioned, and stained with H\&E. For confocal analysis, freshly cut sections were de-

261 parafinized in xylenes and rehydrated. The tissue was blocked using carbohydrate free blocking

262 solution (Vector) and stained with SNA-FITC (Vector) at $0.5 \mu \mathrm{g} / \mathrm{ml}$. Autofluorescence was 263 diminished using TrueVIEW autofluorescence Quenching Kit (Vector) and the slides were 264 mounted using VECTASHILED HardSet Antifade Mounting Medium (Vector). Confocal imaging 265 was performed on a SP5 Laser Scanning Confocal Microscope (Leica).

266 IgG purification

267 IgG was purified by separation over a HiTrap Protein A HP Antibody Purification Column 268 (GE Life Sciences, 17040203) fitted to a GE Life Sciences Akta Purifier 10 HPLC, according to 269 manufacturer instructions. IgG was tittered using an IgG ELISA kit (Bethyl, E90-131) according 270 to manufacturer instructions.

271 Mass spectrometry 
Mass spectrometry N-glycan profile and sialic acid linkage analysis was performed on

273 purified IgG essentially as described previously following DMT-MM and permethylation

274 derivatization (28). IgG samples were lyophilized and first incubated with $2 \mathrm{mg} / \mathrm{mL} 1,4-$

275 dithiothreitol (Millipore-Sigma) $600 \mathrm{mM}$ Tris buffer at $\mathrm{pH} 8.5$ for 1 hour at $50{ }^{\circ} \mathrm{C}$, then $12 \mathrm{mg} / \mathrm{mL}$

276 iodoacetamide (Millipore-Sigma) also in $600 \mathrm{mM}$ Tris at pH 8.5 for 1 hour in the dark. Following

277 dialysis against $50 \mathrm{mM}$ ammonium bicarbonate, samples were dried by lyophilization and

278 resuspended in $50 \mathrm{mM}$ ammonium bicarbonate buffer containing $25 \mu \mathrm{g}$ of TPCK-treated trypsin

279 (Millipore-Sigma) and incubated overnight at $37{ }^{\circ} \mathrm{C}$. The trypsin-digested samples were cleaned

280 using a $50 \mathrm{mg}$ C18 Sep-Pak column (Waters). The lyophilized samples were then resuspended in

$28150 \mathrm{mM}$ ammonium bicarbonate buffer and digested with PNGaseF (New England Biolabs) for a

282 total of 20 hours. The PNGaseF-released N-glycans were isolated on a C18 Sep-Pak column, 283 evenly split into two fractions and dried. solution of $\mathrm{NaOH}$ in DMSO. $500 \mu \mathrm{L}$ of iodomethane (Millipore-Sigma) were added and the

286 samples were mixed vigorously at room temperature for 30 minutes. $1 \mathrm{~mL}$ of MilliQ water was

287 then added to stop the reaction. $1 \mathrm{~mL}$ of chloroform and $3 \mathrm{~mL}$ of MilliQ water were added,

288 vortexed thoroughly, and centrifuged (5000 rpm, $30 \mathrm{sec})$. The aqueous top layer was discarded

289 and $3 \mathrm{~mL}$ of MilliQ water was added to wash the chloroform phase by vortexing and centrifuging

290 as before. This was repeated one additional time before drying the chloroform fraction with a

291 speedvac. The dried permethylated N-glycans were cleaned on a C18 Sep-Pak column and eluted 292 with $3 \mathrm{~mL}$ of $50 \%$ acetonitrile. The eluted fraction was lyophilized prior to MS analysis.

293 For the DMT-MM (4-(4,6-dimethoxy-1,3,5-triazin-2yl)-4-methylmorpholinium chloride)

294 treatment, a solution of 0.5 M of DMT-MM (Millipore-Sigma) was prepared in $500 \mathrm{mM}^{\circ} \mathrm{NH}_{4} \mathrm{Cl}$, 
$\mathrm{pH}$ 6.5. $10 \mu \mathrm{L}$ of the DMT-MM solution were added to the second aliquot of each N-glycan sample

296 and incubated at $60{ }^{\circ} \mathrm{C}$ for 15 hours. Samples were cleaned on a C18 Sep-Pak column, lyophilized,

297 and permethylated as before.

298 Permethylated N-glycans +/- DMT-MM derivatization were dissolved in $10 \mu \mathrm{L}$ of $75 \%$

299 methanol, from which $1 \mu \mathrm{L}$ was mixed with $1 \mu \mathrm{L}$ 2,5-dihydroxybenzoic acid (DHB) $(5 \mathrm{mg} / \mathrm{mL}$ in

$30050 \%$ acetonitrile with $0.1 \%$ trifluoroacetic acid) and spotted on a MALDI polished steel target

301 plate (Bruker Daltonics). MS data were acquired on a Bruker UltraFlex II MALDI-TOF Mass

302 Spectrometer instrument. The reflective positive mode was used, and data were recorded between

303500 and $6000 \mathrm{~m} / \mathrm{z}$. For each MS N-glycan profile, the aggregation of 20,000 laser shots or more

304 were considered for data extraction. Only MS signals matching an N-glycan composition were

305 considered for further analysis. Subsequent MS post-data acquisition analysis was made using

306 mMass (33).

\section{Lectin ELISA}

308 High throughput, multiplexed lectin ELISA was performed on whole plasma samples, as

309 has been published $(27,34)$. Briefly, plasma was diluted to $0.5 \mu \mathrm{g} / \mathrm{ml}$ in carbonate coating buffer

$310(100 \mathrm{mM} \mathrm{NaHCO}, 30 \mathrm{mM} \mathrm{NaCO}$, $\mathrm{pH}$ 9.5), pipetted into a 384 well black ELISA plate, and

311 incubated overnight at $4{ }^{\circ} \mathrm{C}$. The plate was blocked with carbohydrate free blocking solution

312 (Vector, SP-5040) for 1 hour at room temperature. Biotinylated lectins were diluted as previously

313 described and incubated on the plate for 1 hour at room temperature. Signal was detected using

314 streptavidin-Eu (Perkin Elmer 1244-360) and enhancement solution (Perkin Elmer, 4001-0010)

315 measured in a Victor V3 1420 multilabel plate reader. 
318 was fractionated using SNA-agarose (Vector) into $\mathrm{SNA}^{-}$fraction, then washed 3 times (10 mM

319 HEPES, $0.1 \% \mathrm{TX}-100, \mathrm{pH} 7.5)$ and an $\mathrm{SNA}^{+}$fraction was eluted (100 mM glycine, $100 \mathrm{mM}$

320 sodium acetate, $5 \mathrm{mM} \mathrm{MgCl} 2, \mathrm{pH} 4.5)$. IgG concentration was determined as detailed above.

A 96 well ELISA plate was coated with bovine fetuin $(5 \mu \mathrm{g} / \mathrm{mL}$; Sigma $)$ in carbonate buffer

323 overnight, then blocked for 1 hour at room temperature with Carbohydrate-Free blocking solution

324 (Vector Labs), and treated with $0.005 \mathrm{U}$ of neuraminidase from C. perfringens (Sigma) per well

325 in $1 \mathrm{mM} \mathrm{Na}_{2} \mathrm{HPO}_{4}, 1 \mathrm{mM} \mathrm{K}_{2} \mathrm{HPO}_{4}$, and $10 \mathrm{mM} \mathrm{KCl}$ at $\mathrm{pH} 6.0$ for 1 hour at $37^{\circ} \mathrm{C}$. Using harvested

326 mouse plasma, an enzymatic reaction was performed at $37^{\circ} \mathrm{C}$ for 3 hours with or without $100 \mu \mathrm{M}$

327 CMP-sialic acid donor nucleotide-sugar. Added $\alpha 2,6$-linked sialic acids were probed using SNA-

328 biotin $(0.4 \mu \mathrm{g} / \mathrm{mL})$, Eu-conjugated streptavidin (Perkin-Elmer) at $0.1 \mu \mathrm{g} / \mathrm{mL}$, and detected on a

329 Victor V3 multilabel plate reader as previously published (16).

Mice were injecting in the tail vein with $200 \mu \mathrm{g}$ of CMP-SA suspended in PBS and plasma

332 harvested after 24 hours.

335 function "prcomp" was used to calculate principal component analysis. PCA was then blotted 336 using the "fviz_pca_biplot" function. Statistical testing was done by student's T test: *, p $<0.01$ : 


\section{$\underline{\text { References Cited }}$}

339 1. Cobb, B. A. (2020) The history of IgG glycosylation and where we are now. Glycobiology 30, 202-213

2. de Haan, N., Reiding, K. R., Kristic, J., Hipgrave Ederveen, A. L., Lauc, G., and Wuhrer, M. (2017) The N-Glycosylation of Mouse Immunoglobulin G (IgG)-Fragment Crystallizable Differs Between IgG Subclasses and Strains. Front Immunol 8, 608

\section{Kaneko, Y., Nimmerjahn, F., and Ravetch, J. V. (2006) Anti-inflammatory activity of} immunoglobulin $\mathrm{G}$ resulting from Fc sialylation. Science 313, 670-673

4. Rook, G. A., Steele, J., Brealey, R., Whyte, A., Isenberg, D., Sumar, N., Nelson, J. L., Bodman, K. B., Young, A., Roitt, I. M., and et al. (1991) Changes in IgG glycoform levels are associated with remission of arthritis during pregnancy. J Autoimmun 4, 779-794

5. van de Geijn, F. E., Wuhrer, M., Selman, M. H., Willemsen, S. P., de Man, Y. A., Deelder, A. M., Hazes, J. M., and Dolhain, R. J. (2009) Immunoglobulin G galactosylation and sialylation are associated with pregnancy-induced improvement of rheumatoid arthritis and the postpartum flare: results from a large prospective cohort study. Arthritis Res Ther 11, R193

6. Kawahara, J. Y., Irvine, E. B., and Alter, G. (2019) A Case for Antibodies as Mechanistic Correlates of Immunity in Tuberculosis. Front Immunol 10, 996

7. Lofano, G., Gorman, M. J., Yousif, A. S., Yu, W. H., Fox, J. M., Dugast, A. S., Ackerman, M. E., Suscovich, T. J., Weiner, J., Barouch, D., Streeck, H., Little, S., Smith, D., Richman, D., Lauffenburger, D., Walker, B. D., Diamond, M. S., and Alter, G. (2018) Antigenspecific antibody Fc glycosylation enhances humoral immunity via the recruitment of complement. Sci Immunol 3

8. Butler, A. L., Fischinger, S., and Alter, G. (2019) The Antibodiome-Mapping the Humoral Immune Response to HIV. Curr HIV/AIDS Rep 16, 169-179

9. Keser, T., Gornik, I., Vuckovic, F., Selak, N., Pavic, T., Lukic, E., Gudelj, I., Gasparovic, H., Biocina, B., Tilin, T., Wennerstrom, A., Mannisto, S., Salomaa, V., Havulinna, A., Wang, W., Wilson, J. F., Chaturvedi, N., Perola, M., Campbell, H., Lauc, G., and Gornik, O. (2017) Increased plasma N-glycome complexity is associated with higher risk of type 2 diabetes. Diabetologia 60, 2352-2360

10. Barrios, C., Zierer, J., Gudelj, I., Stambuk, J., Ugrina, I., Rodriguez, E., Soler, M. J., Pavic, T., Simurina, M., Keser, T., Pucic-Bakovic, M., Mangino, M., Pascual, J., Spector, T. D., Lauc, G., and Menni, C. (2016) Glycosylation Profile of IgG in Moderate Kidney Dysfunction. J Am Soc Nephrol 27, 933-941

11. Vuckovic, F., Kristic, J., Gudelj, I., Teruel, M., Keser, T., Pezer, M., Pucic-Bakovic, M., Stambuk, J., Trbojevic-Akmacic, I., Barrios, C., Pavic, T., Menni, C., Wang, Y., Zhou, Y., Cui, L., Song, H., Zeng, Q., Guo, X., Pons-Estel, B. A., McKeigue, P., Leslie Patrick, A., Gornik, O., Spector, T. D., Harjacek, M., Alarcon-Riquelme, M., Molokhia, M., Wang, W., and Lauc, G. (2015) Association of systemic lupus erythematosus with decreased immunosuppressive potential of the IgG glycome. Arthritis Rheumatol 67, 2978-2989

12. Anthony, R. M., Wermeling, F., Karlsson, M. C., and Ravetch, J. V. (2008) Identification of a receptor required for the anti-inflammatory activity of IVIG. Proc Natl Acad Sci US A 105, 19571-19578

13. Anthony, R. M., Kobayashi, T., Wermeling, F., and Ravetch, J. V. (2011) Intravenous gammaglobulin suppresses inflammation through a novel $\mathrm{T}(\mathrm{H}) 2$ pathway. Nature $\mathbf{4 7 5}$, $110-113$ 
14. Yu, X., Vasiljevic, S., Mitchell, D. A., Crispin, M., and Scanlan, C. N. (2013) Dissecting the molecular mechanism of IVIg therapy: the interaction between serum IgG and DCSIGN is independent of antibody glycoform or Fc domain. J Mol Biol 425, 1253-1258

15. Temming, A. R., Dekkers, G., van de Bovenkamp, F. S., Plomp, H. R., Bentlage, A. E. H., Szittner, Z., Derksen, N. I. L., Wuhrer, M., Rispens, T., and Vidarsson, G. (2019) Human DC-SIGN and CD23 do not interact with human IgG. Sci Rep 9, 9995

16. Jones, M. B., Oswald, D. M., Joshi, S., Whiteheart, S. W., Orlando, R., and Cobb, B. A. (2016) B-cell-independent sialylation of IgG. Proc Natl Acad Sci U S A 113, 7207-7212

17. Wang, T. T., Maamary, J., Tan, G. S., Bournazos, S., Davis, C. W., Krammer, F., Schlesinger, S. J., Palese, P., Ahmed, R., and Ravetch, J. V. (2015) Anti-HA Glycoforms Drive B Cell Affinity Selection and Determine Influenza Vaccine Efficacy. Cell 162, 160169

18. Ohmi, Y., Ise, W., Harazono, A., Takakura, D., Fukuyama, H., Baba, Y., Narazaki, M., Shoda, H., Takahashi, N., Ohkawa, Y., Ji, S., Sugiyama, F., Fujio, K., Kumanogoh, A., Yamamoto, K., Kawasaki, N., Kurosaki, T., Takahashi, Y., and Furukawa, K. (2016) Sialylation converts arthritogenic IgG into inhibitors of collagen-induced arthritis. Nat Commun 7, 11205

19. Jones, M. B., Nasirikenari, M., Lugade, A. A., Thanavala, Y., and Lau, J. T. (2012) Antiinflammatory $\operatorname{IgG}$ production requires functional P1 promoter in beta-galactoside alpha2,6-sialyltransferase 1 (ST6Gal-1) gene. J Biol Chem 287, 15365-15370

20. Dalziel, M., Lemaire, S., Ewing, J., Kobayashi, L., and Lau, J. T. (1999) Hepatic acute phase induction of murine beta-galactoside alpha 2,6 sialyltransferase (ST6Gal I) is IL-6 dependent and mediated by elevation of exon $\mathrm{H}$-containing class of transcripts. Glycobiology 9, 1003-1008

21. Kitazume, S., Tachida, Y., Oka, R., Kotani, N., Ogawa, K., Suzuki, M., Dohmae, N., Takio, K., Saido, T. C., and Hashimoto, Y. (2003) Characterization of alpha 2,6-sialyltransferase cleavage by Alzheimer's beta -secretase (BACE1). J Biol Chem 278, 14865-14871

22. Kitazume, S., Suzuki, M., Saido, T. C., and Hashimoto, Y. (2004) Involvement of proteases in glycosyltransferase secretion: Alzheimer's beta-secretase-dependent cleavage and a following processing by an aminopeptidase. Glycoconj J 21, 25-29

23. Appenheimer, M. M., Huang, R. Y., Chandrasekaran, E. V., Dalziel, M., Hu, Y. P., Soloway, P. D., Wuensch, S. A., Matta, K. L., and Lau, J. T. (2003) Biologic contribution of P1 promoter-mediated expression of ST6Gal I sialyltransferase. Glycobiology 13, 591600

24. Wandall, H. H., Rumjantseva, V., Sorensen, A. L., Patel-Hett, S., Josefsson, E. C., Bennett, E. P., Italiano, J. E., Jr., Clausen, H., Hartwig, J. H., and Hoffmeister, K. M. (2012) The origin and function of platelet glycosyltransferases. Blood 120, 626-635

25. Oswald, D. M., Zhou, J. Y., Jones, M. B., and Cobb, B. A. (2020) Disruption of hepatocyte Sialylation drives a T cell-dependent pro-inflammatory immune tone. Glycoconj J 37, 395407

26. Oswald, D. M., Jones, M. B., and Cobb, B. A. (2020) Modulation of hepatocyte sialylation drives spontaneous fatty liver disease and inflammation. Glycobiology 30, 346-359

27. Oswald, D. M., Sim, E. S., Baker, C., Farhan, O., Debanne, S. M., Morris, N. J., Rodriguez, B. G., Jones, M. B., and Cobb, B. A. (2019) Plasma glycomics predict cardiovascular disease in patients with ART-controlled HIV infections. FASEB J 33, 1852-1859 
28. Alley, W. R., Jr., and Novotny, M. V. (2010) Glycomic analysis of sialic acid linkages in glycans derived from blood serum glycoproteins. J Proteome Res 9, 3062-3072

29. Nasirikenari, M., Chandrasekaran, E. V., Matta, K. L., Segal, B. H., Bogner, P. N., Lugade, A. A., Thanavala, Y., Lee, J. J., and Lau, J. T. (2010) Altered eosinophil profile in mice with ST6Gal-1 deficiency: an additional role for ST6Gal-1 generated by the P1 promoter in regulating allergic inflammation. $J$ Leukoc Biol 87, 457-466

30. Nasirikenari, M., Segal, B. H., Ostberg, J. R., Urbasic, A., and Lau, J. T. (2006) Altered granulopoietic profile and exaggerated acute neutrophilic inflammation in mice with targeted deficiency in the sialyltransferase ST6Gal I. Blood 108, 3397-3405

31. Jones, M. B., Nasirikenari, M., Feng, L., Migliore, M. T., Choi, K. S., Kazim, L., and Lau, J. T. (2010) Role for hepatic and circulatory ST6Gal-1 sialyltransferase in regulating myelopoiesis. J Biol Chem 285, 25009-25017

32. Mahan, A. E., Jennewein, M. F., Suscovich, T., Dionne, K., Tedesco, J., Chung, A. W., Streeck, H., Pau, M., Schuitemaker, H., Francis, D., Fast, P., Laufer, D., Walker, B. D., Baden, L., Barouch, D. H., and Alter, G. (2016) Antigen-Specific Antibody Glycosylation Is Regulated via Vaccination. PLoS Pathog 12, e1005456

33. Strohalm, M., Kavan, D., Novak, P., Volny, M., and Havlicek, V. (2010) mMass 3: a crossplatform software environment for precise analysis of mass spectrometric data. Anal Chem 82, 4648-4651

34. Oswald, D. M., and Cobb, B. A. (2018) Emerging glycobiology tools: A renaissance in accessibility. Cell Immunol 333, 2-8 
bioRxiv preprint doi: https://doi.org/10.1101/2022.02.01.478679; this version posted February 4, 2022. The copyright holder for this preprint (which was not certified by peer review) is the author/funder. All rights reserved. No reuse allowed without permission.

\section{Acknowledgements}

452 The authors wish to acknowledge Dr. Mark B. Jones for scientific input in the early phases of this

453 project, and for the BACE-1 knockout IgG analysis. Funding for this work was through grants to

454 BAC from the NIH (GM115234 and AI154899) and to JYZ and DMO from the NIH (AI089474). 
bioRxiv preprint doi: https://doi.org/10.1101/2022.02.01.478679; this version posted February 4, 2022. The copyright holder for this preprint (which was not certified by peer review) is the author/funder. All rights reserved. No reuse allowed without permission.

\section{Conflict of interest}

456 The authors declare that they have no financial conflicts of interest with the contents of this article. 
bioRxiv preprint doi: https://doi.org/10.1101/2022.02.01.478679; this version posted February 4, 2022. The copyright holder for this preprint (which was not certified by peer review) is the author/funder. All rights reserved. No reuse allowed without permission.

\section{Author Contributions}

458 DMO, experimental design, data collection and analysis, manuscript writing; JYZ and LMG, data

459 acquisition; SDL and RDC, mass spectrometry design, data collection and analysis, manuscript

460 editing; BAC, experimental design, data analysis, manuscript writing, funding. 


\section{Abbreviations}

462 4-(4,6-dimethoxy-1,3,5-triazin-2yl)-4-methylmorpholinium chloride (DMT-MM)

463 B cell-specific conditional knockout of ST6Gall (BcKO)

464 cytidine monophosphate $\mathrm{N}$-acetylneuraminic acid (CMP-sialic acid)

465 hepatocyte-specific conditional knockout of ST6Gal1 (HcKO)

466 intravenous immunoglobulin therapy (IVIg)

467 mouse lacking ST6Gal1 in both the hepatocyte and B cell compartments (BHcKO)

468 principle component analysis (PCA)

469 rheumatoid arthritis (RA)

$470 \quad$ wild type (WT) 


\section{Figure Legends}

472 Figure 1. Plasma glycoprotein glycan changes are associated with both hepatocyte ST6Gal1 and immunization

474 (A) Lectin ELISA of total plasma glycoproteins in resting and immunized WT and HcKO mice 2 475 weeks after the final booster exposure using an array of lectins revealed a loss of SNA in HcKO 476 samples, and reductions in ConA, SNA, PHA-E, DSL, PHA-L, RCA120, and WGA in WT and 477 HcKO associated with immunization. Increased LCA and AAL was also seen in both strains 478 following immunization. (B) PCA results demonstrated that immunized mouse plasma glycans are 479 more similar to each other than either naïve mouse. PC1 and PC2 scores for each of the four groups 480 show the dominance of immunization in determining overall plasma glycan characteristics. $\mathrm{N}=3$ 481 for each condition and strain.

Figure 2. IgG glycan sialylation is consistent between WT and HcKO mice

483 (A) IgG titers in immunized and non-immunized mice, 2 weeks following the final immunization, 484 showed strong IgG responses in both strains, despite a slightly reduced overall response in HcKO 485 mice. $\mathrm{N}=3$ (B) Lectin ELISA data on purified IgG revealed a lack of difference in $\alpha 2,6$-sialylation 486 between WT and HcKO IgG. N=4-5 (C) Based on mass spectrometry, total IgG sialylated glycan 487 species were increased in HcKO mice. $\mathrm{N}=3$ (D) No difference in linkage distribution of sialic acids 488 was detected. $\mathrm{N}=3$ ( $\mathrm{E}$ and F) A breakdown of individual sialylated glycan species found by mass 489 spectrometry showed that the distribution of mono-sialylated, di-sialylated, Neu5Ac, Neu5Gc, and 490 fucosylation was indistinguishable between WT and $\mathrm{HcKO}$ mice. $\mathrm{N}=3$ 
(A) BACE-1 knockout mice carried modestly but significantly higher IgG titers compared to

493 similarly housed WT control mice. $\mathrm{N}=12$ (B) The degree of $\alpha 2,6$-sialylation of IgG from resting

494 and non-immunized WT and BACE-1 knockout mice, as measured by SNA, was indistinguishable.

$495 \mathrm{~N}=4$ (C) Mice with germline knockout of ST6Gal1 lacked detectable IgG $\alpha 2,6$-sialylation. N=6-9

\section{Figure 4. Creation and general characterization of the BHcKO mouse}

497 (A) Scheme, genetics, and validating genotype analysis of the dual-deficient mutant mice. (B)

498 Confocal staining for SNA in the liver revealed the expected deficiency of sialylation in HcKO

499 and $\mathrm{BHcKO}$, but not WT and BcKO tissue; image border color description is indicated in panel A.

500 (C) SNA and ECL lectin flow cytometry analysis of blood CD19 ${ }^{+}$B cells demonstrated the 501 ablation of surface $\alpha 2,6$ sialic acids and increase in terminal galactose respectively on $\mathrm{BcKO}$ and 502 BHcKO mice cells, but not in WT and HcKO. N=3-5

\section{Figure 5. Plasma ST6Gal1 activity is lost in HcKO and BHcKO mice}

504 (A) Addition of $\alpha 2,6$ sialic acids to plate-bound neuraminidase (NA)-treated fetuin with plasma

505 from $\mathrm{WT}, \mathrm{BcKO}, \mathrm{HcKO}$, and $\mathrm{BHcKO}$ mice, and with or without added CMP-sialic acid 506 nucleotide-sugar donor, showing a loss of detectable activity in HcKO and BHcKO plasma. N=12

507 (B) I.v. injection of CMP-SA did not alter the total IgG concentration, but led to an increase in $508 \alpha 2,6$-sialylation in WT mice, confirming the function of plasma-localized ST6Gal1. N=6-9

\section{Figure 6. BHcKO IgG glycans mirror WT glycans}

510 (A) IgG titers in immunized and non-immunized mice revealed slightly lower overall IgG titers in 511 all three ST6Gal1 knockout mice. N=4 (B) With the exception of the BcKO strain, the Ig class and 512 subclass distribution is essentially unchanged across strains. BcKO mice showed a decrease in 513 IgG1 and IgG3 and an increase in IgG2a and IgG2b. N=3 (C) Based on mass spectrometry, overall 
514 IgG sialylation of IgG glycans 8 weeks post immunization was indistinguishable between strains.

$515 \mathrm{~N}=3$ (D) Likewise, the distribution of $\alpha 2,3$ and $\alpha 2,6$ linkages were indistinguishable. (E and F) A

516 breakdown of specific sialylated glycan species found showed that the general distribution of

517 mono-sialylated, di-sialylated, Neu5Ac, Neu5Gc, and fucosylation is the same in all strains of 518 mice, although G2FS2 structures, particularly with two Neu5Gc residues were higher in WT and

519 HcKO compared to $\mathrm{BcKO}$ and $\mathrm{BHcKO}$ samples. $\mathrm{N}=3$

Figure 7. IgG sialylation linkage distribution is distinct for each sialylated species

521 (A) Comparison of the distribution of $\alpha 2,3$ and $\alpha 2,6$ linkages on a singly sialylated glycan species-

522 by-species level at 2 weeks of WT and HcKO mice and 8 weeks of WT, HcKO, BcKO, and

523 BHcKO mice. (B) Comparison of the distribution of $\alpha 2,3$ and $\alpha 2,6$ linkages on a dually sialylated

524 glycan species-by-species level at 2 weeks of WT and HcKO mice and 8 weeks of WT, HcKO,

$525 \mathrm{BcKO}$, and $\mathrm{BHcKO}$ mice. The data revealed that linkage distributions change differentially among

526 sialylated species. All data $\mathrm{N}=3$

\section{Figure 8. IgG sialylation increases over time following immunization.}

528 Time course comparison of the main singly and dually sialylated glycan species in WT and HcKO

529 mice at 2 and 8 weeks post final immunization boost, which indicated a shift towards structures

530 containing two sialic acids at a longer time point after the most recent exposure to antigen. $\mathrm{N}=3$ 


\section{Figure 1}

A
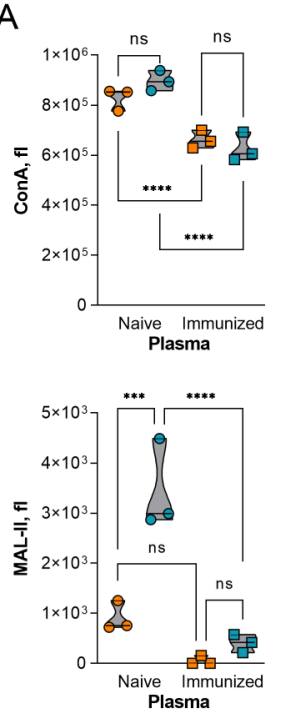
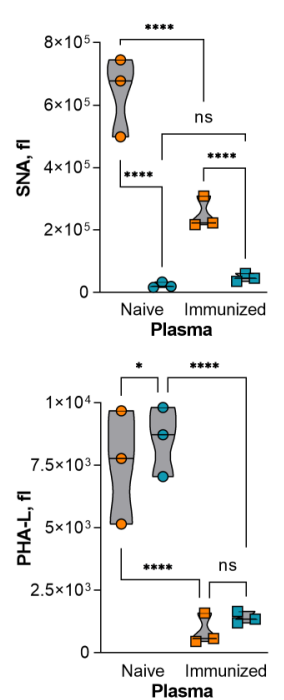
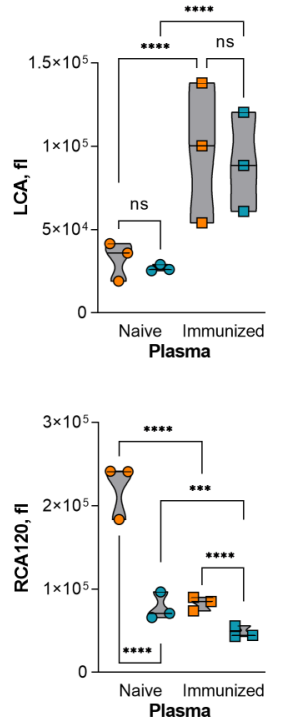
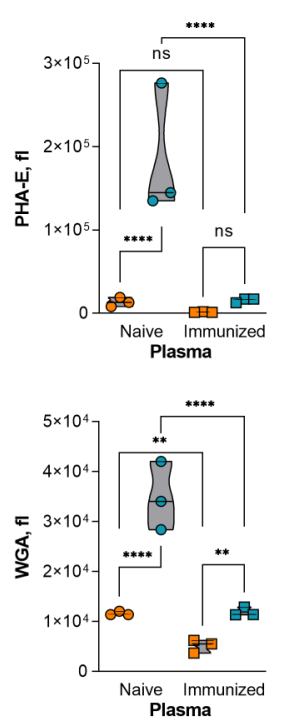

B

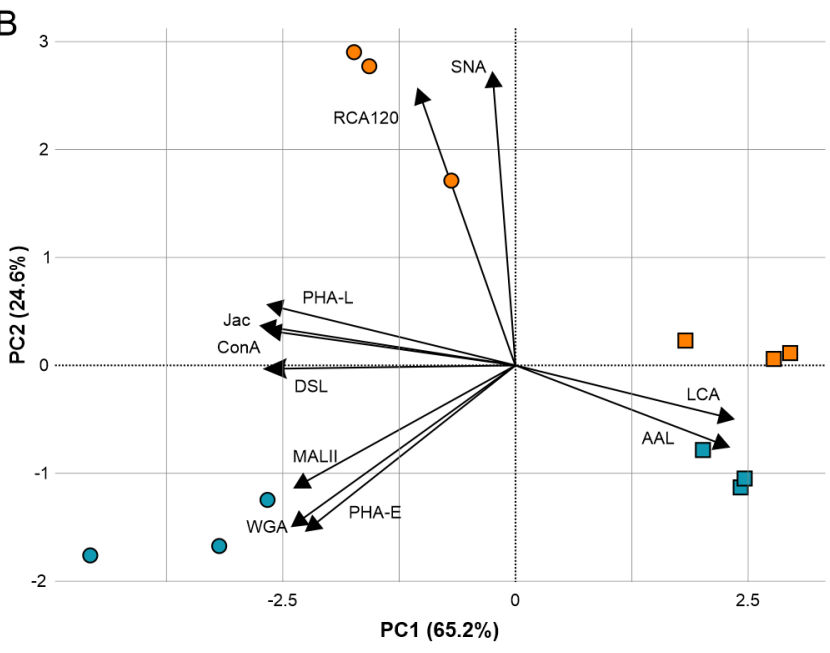

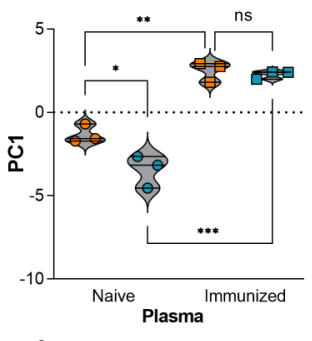

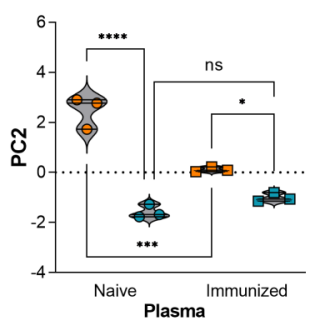

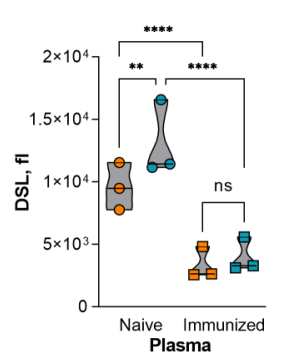

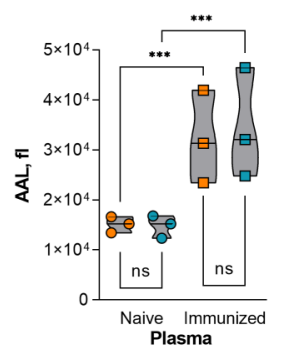

O Naive ST6"

O Naive HcKO

$\square$ Immunized ST6"

口 Immunized HcKO 
Figure 2
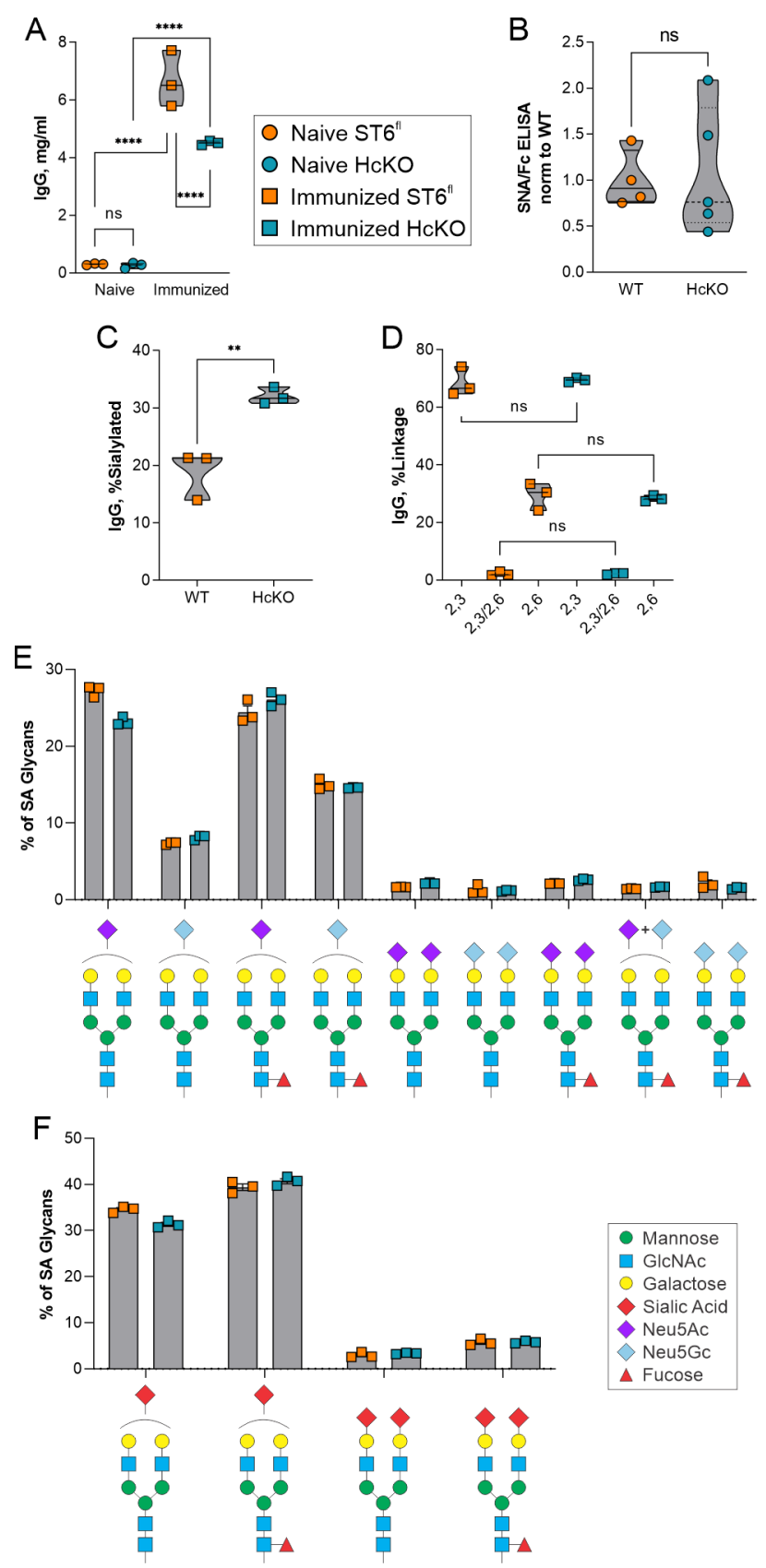

- Mannose - GICNAc - Galactose

Sialic Acid

Neu5AC

$\triangle$ Fucose 
bioRxiv preprint doi: https://doi.org/10.1101/2022.02.01.478679; this version posted February 4, 2022. The copyright holder for this preprint (which was not certified by peer review) is the author/funder. All rights reserved. No reuse allowed without permission.

Figure 3
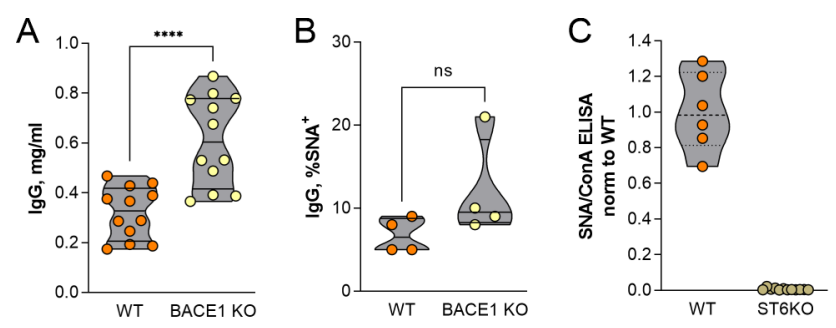


\section{Figure 4}
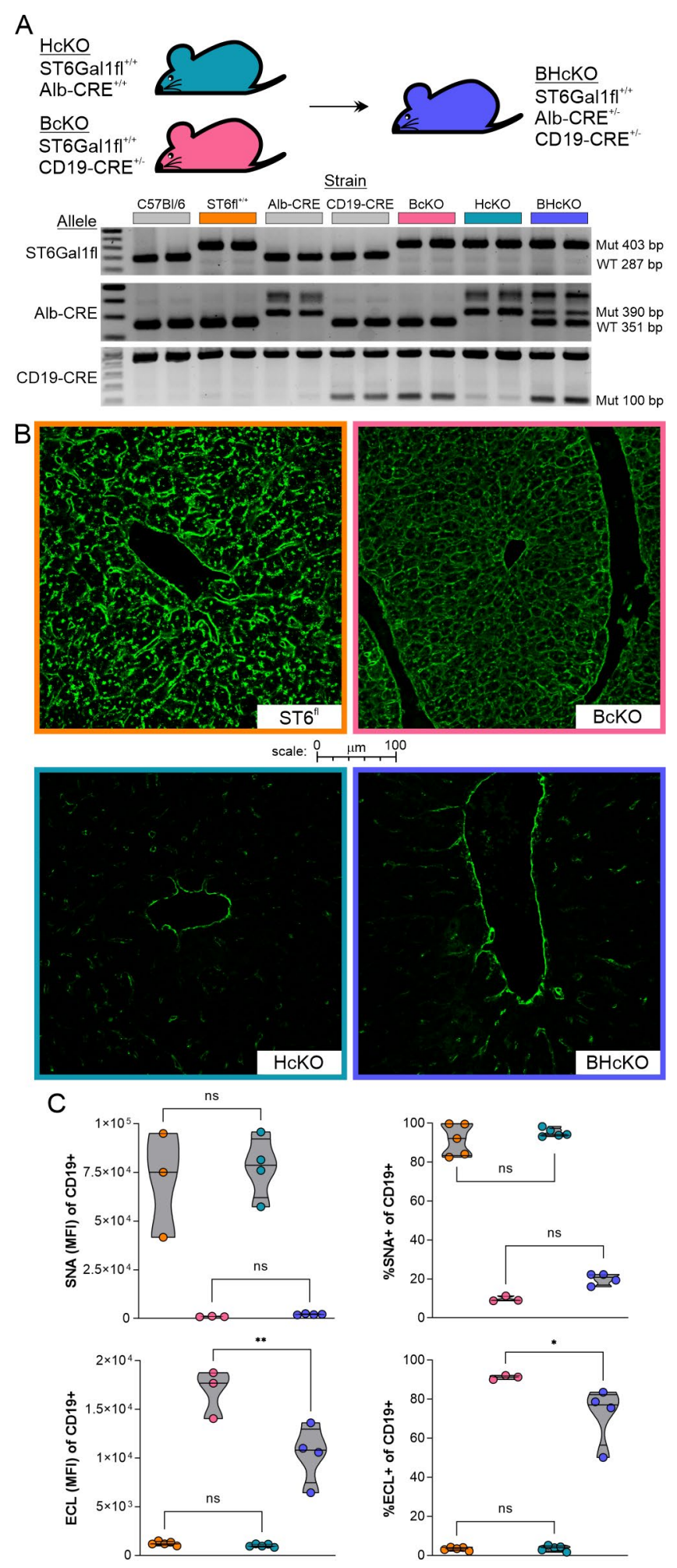

O Naive ST6" O Naive BcKO O Naive HcKO O Naive BHcKO 


\section{Figure 5}
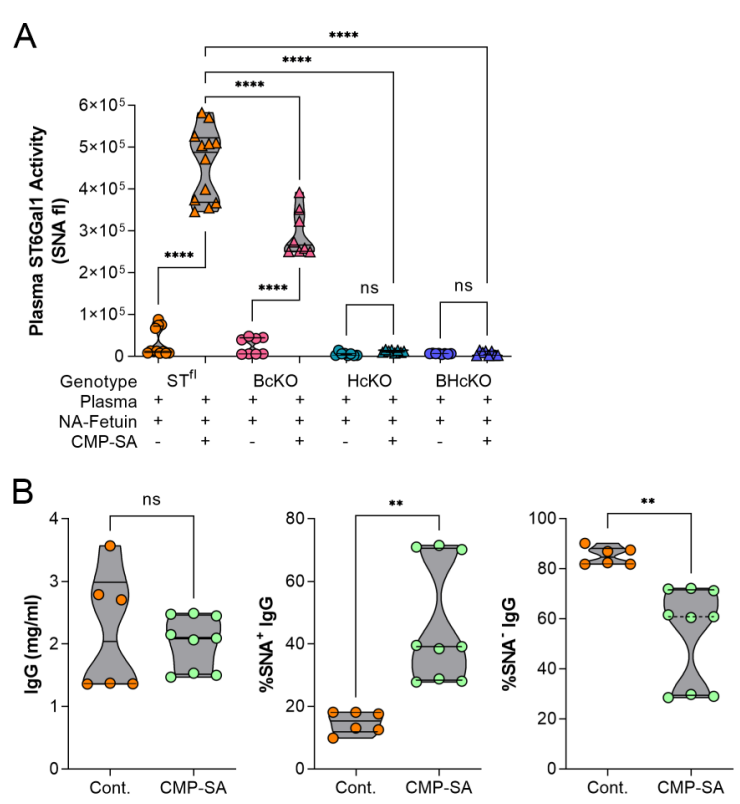


\section{Figure 6}
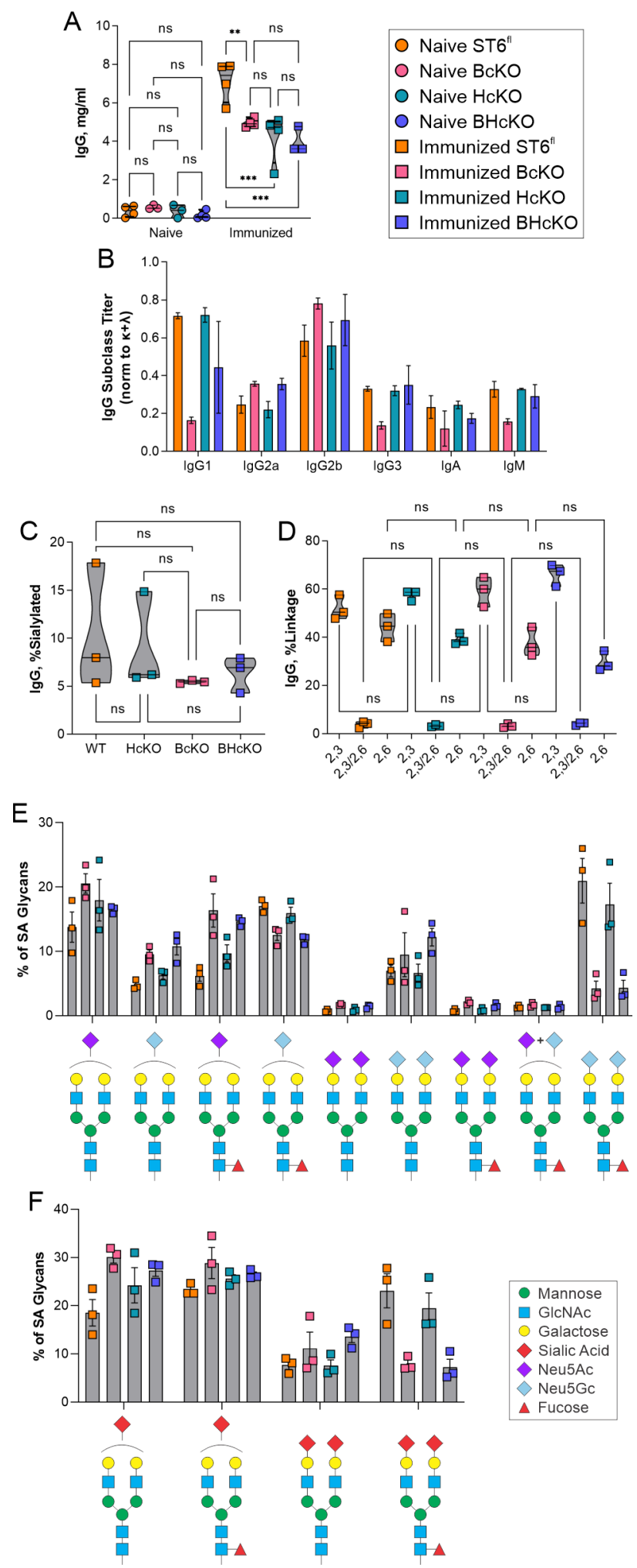
bioRxiv preprint doi: https://doi.org/10.1101/2022.02.01.478679; this version posted February 4, 2022. The copyright holder for this preprint (which was not certified by peer review) is the author/funder. All rights reserved. No reuse allowed without permission.

\section{Figure 7}

A
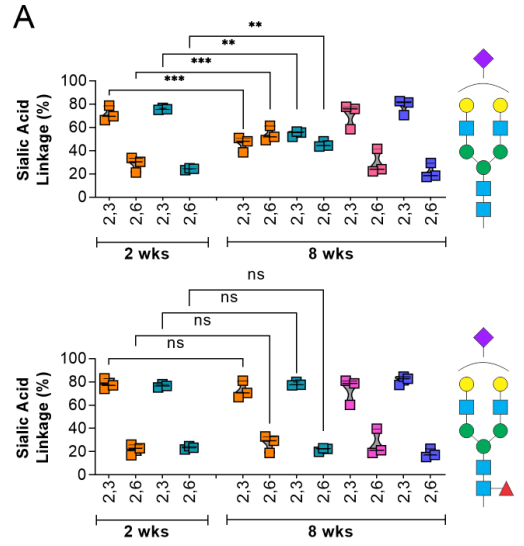

B
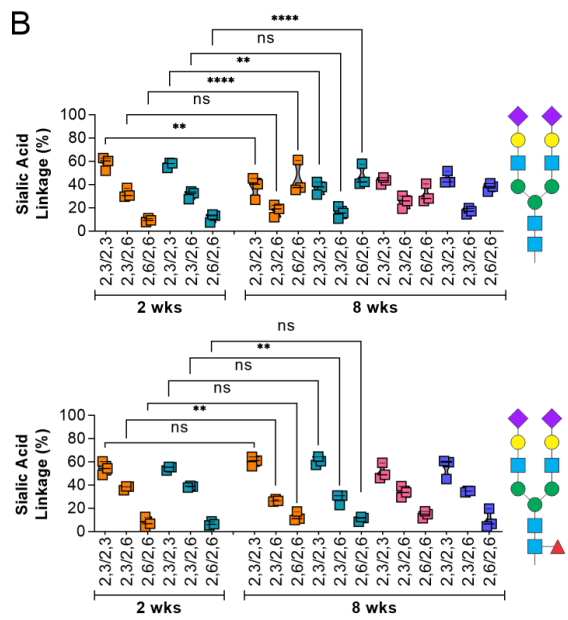
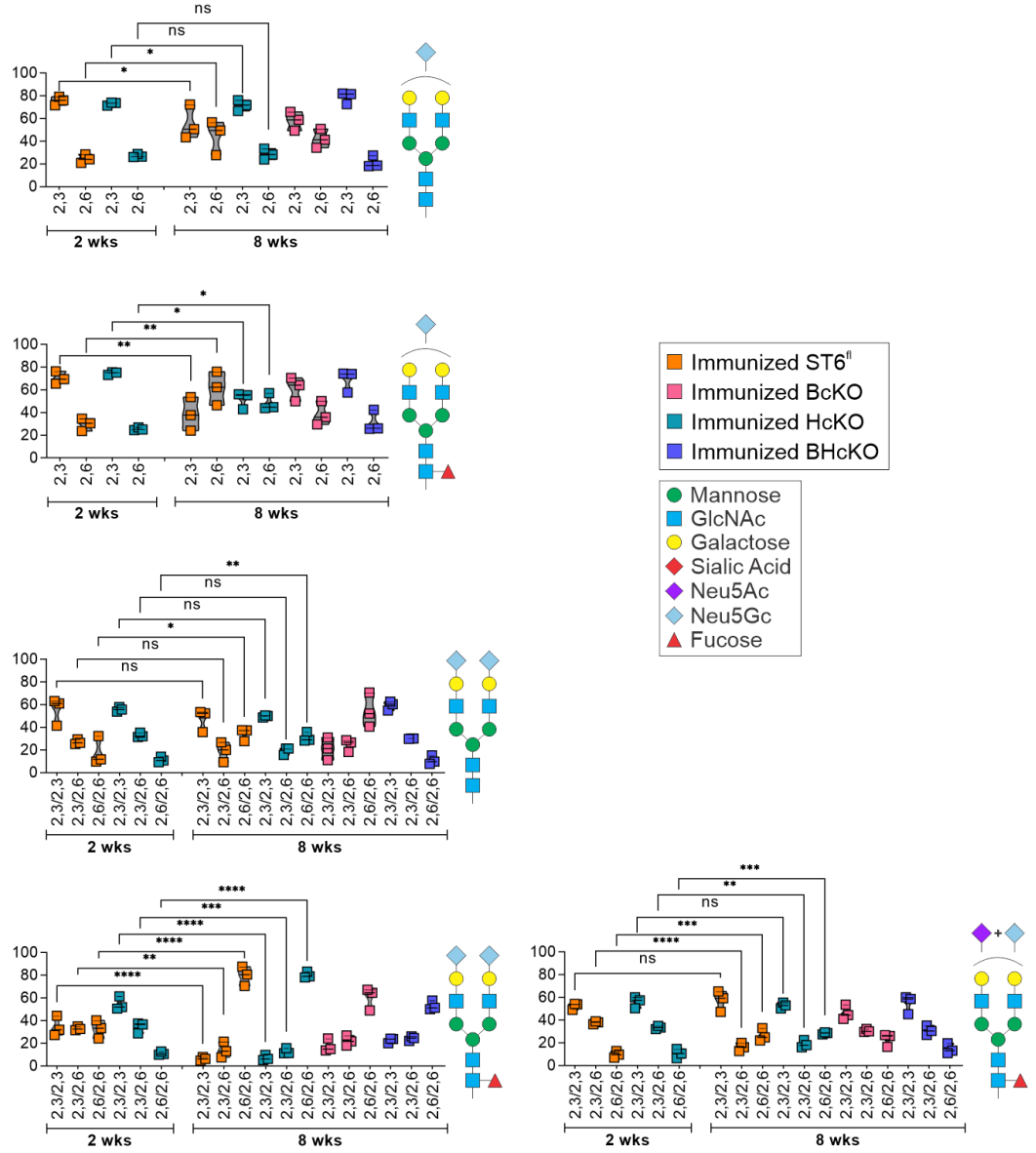
bioRxiv preprint doi: https://doi.org/10.1101/2022.02.01.478679; this version posted February 4, 2022. The copyright holder for this preprint (which was not certified by peer review) is the author/funder. All rights reserved. No reuse allowed without permission.

Figure 8
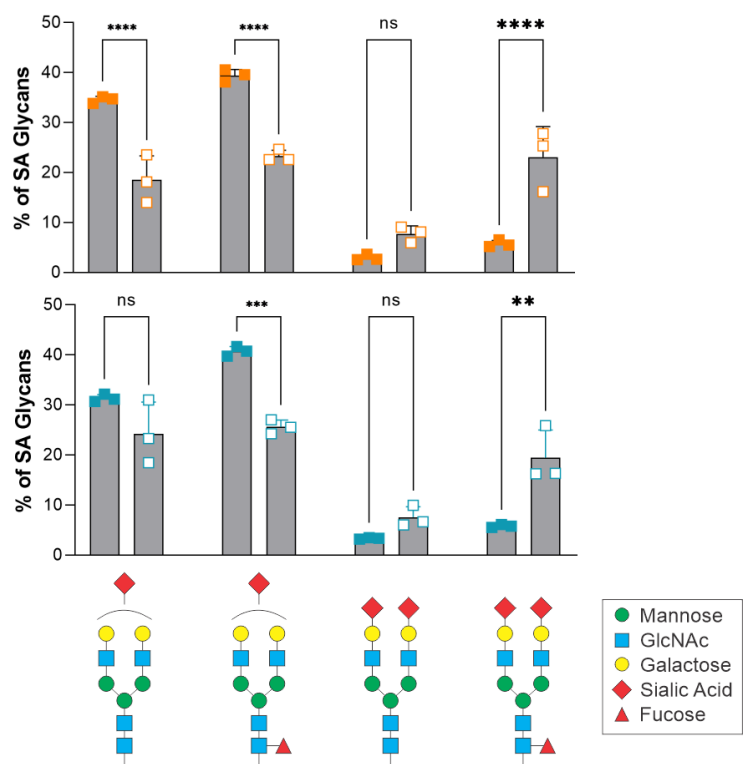

Immunized ST6" 2 wks post $\square$ Immunized ST6 8 wks post
Immunized HcKO 2 wks post $\square$ Immunized HcKO 8 wks post 